\title{
INTERVIEW WITH SPANISH LANGUAGE DEPARTMENT. DIRECTORATE-GENERAL FOR TrANSLATION (DGT) EUROPEAN COMMISSION. JOSÉ LuIS VEGa (HEAd OF DEPARTMENT); AlBERTO Rivas (QUALITY OFFICER) AND LUIS GONZÁLEZ (TERMINOLOGIST) By Carmen Valero-Garcés
}

The Directorate General for Translation (DGT) of the European
Commission translates written texts into and out of the European
Union's 24 official languages and, occasionally, also from some
non-official languages. It pursues the usage of appropriate
terminology in all official languages and it provides linguistic
advice to other Directorate-Generals and European Commission
services.
Regarding its organization, there are four Directorates for
Translation (A, B, C, and D), a Directorate for Resources (R) and a
Directorate for Customer Relations (S). In turn, the Directorates of
Translation are divided into different linguistic departments so as to
cover all official languages. One of them is the Spanish Language
Department, which comprises two translation units split over
Brussels and Luxembourg, respectively. This department lays down
the rules regarding linguistic usage, as well as the criteria for
translating documents for both in-house and freelance translators that
receive translation assignments from the DGT. The work carried out
by these linguistic departments allows the promotion of
multilingualism in the European Union and assures that citizens can
have access to European Union legislation in their own language.

\section{What role does the use of ICTs play in translation in the EU?}

DGT is an intense user of specialised IT applications. Computer assisted translation tools and linguistic databases are an integral part of DGT's differentiated resource mix (in-house translators, free-lance translators, tools). They contribute to the efficient delivery of high quality services to DGT's customers. More specifically the IT systems developed, used and maintained by DGT serve the following main purposes:

- To provide translators, editors and terminologists with efficient tools to support accuracy, consistency and efficiency in their work. This includes computer-assisted translation tools, automated pre-processing information systems and machine 
translation as well as easy access to high quality corpora, data repositories, terminological and documentary resources.

- To provide managers with efficient tools to manage work allocation and monitor deliveries.

- To provide administrative staff with efficient tools allowing them to manage demand and allowing DGT to outsource part of the production, thus matching supply and demand.

- To provide external translators with efficient tools to cooperate and communicate with DGT.

- To provide all translation services of the EU institutions with access to common tools developed by DGT, thus obtaining economies of scale and more consistency in the translations delivered.

- To provide machine translation services to EU institutions and public administrations and online services in the EU Member States.

\section{Which are the most commonly used tools? Which is the place of machine translation (MT)?}

Beyond the workflow applications, EURAMIS, IATE and the computer assisted translation (CAT) tool (SDL Studio 2015) remain the main computer tool for DGT translators. We have recently migrated to the latest version of our CAT tool, which is integrated into a large CAT environment. "Modularity", "interoperability" and "flexibility" are key concepts in our IT development.

EURAMIS (European advanced multilingual information system) is a system of databases or memories (and associated tools) managed by the EU institutions jointly or individually. More than a memory, it is a multilingual repository of equivalent strings of texts, phrases ("segments") belonging to official EU documents allowing their re-use in translation in all European Institutions. The system also contains a set of interfaces to manage access rights for sentence and document management, based on the user's login identification, for both Commission staff and other institution's staff. This repository of shared translation memories contains most, although not all, of the documents which make up the Acquis communautaire, as well as some other documents which are not part of the Acquis.

IATE (Inter-Active Terminology for Europe) is the EU's inter-institutional terminology database. IATE has been used in the EU institutions and agencies since summer 2004 for the collection, dissemination and shared management of EU-specific terminology. We will talk more in detail later about this resource and its evolution.

eTranslation (the new brand of the former MT@EC) is the EU's machine translation system which serves to automatically translate documents and text snippets in a secure environment that ensures the respect of IPR. The system covers all official EU languages and automatic translation between closely related languages is expected to increase the usefulness of MT output.

eTranslation provides input for the work of translators in DGT and other language services of EU institutions and bodies. The results produced by eTranslation benefit from the fact that the system is built on a vast corpus of EU legislation and other EU documents. Feedback from translators feeds, in addition to that, into concrete quality improvement actions. 
The tool is offered to public administrations in the Member States. It is also the core element of the eTranslation platform building block deployed with funding from the Connecting Europe Facility, CEF (see https://ec.europa.eu/digital-single-market/en/connecting-europefacility) between 2015 and 2020. CEF is part of Digital Single Market, which is one of the ten priorities of the Juncker Commission.

eTranslation, both as an internal tool and as the starting point of the Automated Translation platform, a core building block for the Connecting Europe Facility (CEF) ecosystem of public online services, will remain a flagship project.

\section{Quality being one of the fundamental principles, how is the quality of machine translation controlled?}

In any case machine translation at DGT is a basic and auxiliary tool, fully integrated into our CAT tools. Given the inherent risk of errors, raw machine translations have therefore to be post-edited and double-checked, according to our quality standards.

Quality is of paramount importance to us, for obvious reasons of legal certainty and accountability. In line with the principles defined in the DGT Quality Management Framework, the quality management team is formed by four quality managers, one per each ABCD directorate, and is split over two sites: Brussels (Dir A and D) and Luxembourg (Dir B and C). The DGT Quality management framework defines the key concepts and principles for quality management and outlines the structure of quality management-related work, with its main actors and processes. DGT Translation quality guidelines provide guidance on translation, quality control, risk assessment and divide our texts into four categories according to their purpose. Our quality coordinators have also drafted quick reference cards on translation and revision, risk assessment, and risk mitigation which provide a succinct version of the guidelines.

In parallel with the internal quality control we have drafted the DGT Guidelines for evaluation of outsourced translations, describing the process of evaluation, marking and quality control of outsourced translations.

In order to set up quality benchmarks or references on contents, DGT language Departments have language-specific style guides and guidelines. At the Spanish Department, we have the Guía del Departamento, which needs some updating yet but provides our translators and freelancers with very useful information.

Quality managers meet weekly to coordinate and discuss the state of play of quality actions, bring up issues, agree on a course of action and exchange information. As a rule, their meetings are held via MOVI, and on some issues via videoconference. Regular face-to-face meetings are organised to ensure smooth cooperation.

Quality managers hold face-to-face meetings with the quality officers (QOs) of the language departments twice a year. They also have informal coffees with them (separately in Brussels and Luxembourg) to discuss ongoing issues and share information. Quality managers also organise once/twice a year team events with language departments' validators / freelance correspondents to discuss issues related to outsourcing. 
In our Quality management framework, quality is defined as "compliance with specifications, or fitness-for-purpose". This framework identifies the core processes for quality assurance.

DGT assigns translation and revision tasks on the basis of risk assessments where the purpose of the documents and the competence profiles of the staff are taken into account. On the basis of the risks and purposes involved, the texts are grouped into four categories:

1) Legal documents;

2) Policy and administrative documents;

3) Information for the public; and

4) Incoming documents.

All translation work should comply with the general principles and quality requirements for professional translation laid down in the international standard ISO 17100.

\section{Has the massive use of TM or CAT tools led to a change in the translator's profile? Could you point out some new elements that you think should be included in the training of the EU translator?}

It is indisputable that the translator's environment has become digital. Millennials are naturally familiar with technology; therefore we have no problems with the digital competence of our young translators, since they have already received the necessary training at the university.

As we said, besides the commercial tools, DGT has developed a number of in-house applications to handle the management and the workflow of the translation requests and the related documents, and to support the work of the translators and other business. Therefore we provide internal training on these applications and workflow to the end users such as CatTool/Studio - Tradesk (translators' desktop) - DGTVista - Mandesk - Poetry - Euramis LegisWrite - E-Greffe, etc. Newcomers are invited to attend these training courses as a part of their obligatory training path. Besides, as any other big organization, we have our own Knowledge Management Strategy.

In conclusion, basic computer literacy is not enough for translators who want to take advantage of new job offers related to the translation and localization market: project manager, linguistic application developer, localization specialist. Universities should offer adequate training to meet these needs.

\section{As IATE is a tool with a long tradition in the EU, could you give a brief definition and indicate how it has evolved since its creation?}

IATE (Inter-Active Terminology for Europe) is a dynamic database designed to support the multilingual drafting of EU texts, and legal texts in particular. It aims to provide relevant, reliable, easily accessible data which represent a distinct added value compared with other sources of lexical information (e.g. translation memories, the internet, electronic archives). 
For IATE to be usable with translation aid software, the database must be managed with rigour: such software is able to retrieve information from IATE, but, unlike translators, is not able to detect discrepancies between language versions.

Recently our database is being progressively integrated into our CAT environment tools. New IATE extracts provided by our central terminology unit for direct use in SDL Studio are available on a shared drive and we are also developing specific databases on some topics (such as economy and finance, migration, etc.) or for the translation of some specific packages of documents where terminological harmonization is very necessary from the start.

Currently the IATE 2 Task Force, created by the IATE Management Group, is replacing the current version and user interfaces by a state-of-the art solution that is better adapted to the working methods of the IATE partners. In practice we are going further and, beyond the simple improvement of data entry, we are about to see the birth of a new and fully redeveloped IATE with new features: task management screen (integration of terminology projects management and verification of ongoing work items), user dashboard and other reinforced communication features. The new revamped IATE interface is being tested and the completion of the IATE 2 project is expected later this year.

\section{Since terminology is the foundation of IATE, can you tell us what changes have happened in the treatment and use of IATE in the last decade?}

First of all, we have to say that multilingual terminology makes a great contribution to the EU policy of encouraging multilingualism and making EU legislation more transparent to the citizen. In our opinion the key words in regards to the evolution of terminology are: authority, validation, interactivity, networks, linked data, dissemination and access to knowledge.

Terminology on the web allows us to develop the full potential of interactivity. The fact that IATE is available to the public gives freelance translators access to validated terminology and also allows terminologists to receive feedback from external users.

Since translators are always looking for authority, we (terminologists) need to focus on validation and quality issues. For this reason the Spanish language Department has been supporting the Terminesp project from the very beginning and is interested in its development. This platform will allow us to access to validated terminology and, at the same time, will ease the spread of our own terminology resources. We are immersed by a huge mass of information, Nevertheless it's not quantity, but quality that matters. Ideally, it would be even possible to consider the direct participation of specialists in the validation of terms or terminological collections, which will increase the reliability of our terminological data and, therefore, the consistency of our translations.

On the other hand, the terminological databases will become over time (in fact, they are already becoming) repositories of knowledge, in which the terms may serve rather as an index or a catalogue granting access to a huge amount of specialized resources. We will soon see what the future holds for us. 


\section{Apart from ICTs and technological advances in communication, there are also other changes taking place in societies as they evolve towards multicultural societies and with movements of migrant and refugee populations with their languages and cultures, how have these changes in EU language policy and in particular in DGT affected or may affect?}

The EU is founded on the 'united in diversity' principle: diversity of cultures, customs, beliefs - and languages. Article 22 of the Charter of Fundamental Rights of the European Union, proclaimed in 2000 and amended in 2007, requires the EU to respect cultural, religious and linguistic diversity. Article 21 prohibits various forms of discrimination, including on language. This principle applies not only to the official languages of the EU, but also to the many regional and minority languages spoken by certain population groups. Together with respect for the individual, openness to other cultures, tolerance and acceptance of others, respect for linguistic diversity are core EU values.

The Treaty on the Functioning of the European Union guarantees the freedoms and rights enshrined in the Charter of Fundamental Rights of the European Union and gives the Charter binding legal force.

Unlike other international organisations which work in fewer languages, the EU institutions are themselves a level of government. As such, they pass laws that are directly binding on EU citizens. As a matter of simple natural justice, EU citizens - and EU courts - must be able to understand the laws they have to obey or enforce. As a democratic institution, the European Commission has to communicate with EU citizens, national governments and civil services, businesses and other organisations all over the EU in their languages. The public have a right to know what is being done in their name and must be able to play an active part.

The legal basis for multilingualism at the EU are articles 20 and 24 of the Treaty on the Functioning of the European Union (TFEU) and Council Regulation 1 of 1958 determining the languages to be used by the European Economic Community:

'The official languages and the working languages of the institutions of the Union shall be Bulgarian, Croatian, Czech, Danish, Dutch, English, Estonian, Finnish, French, German, Greek, Hungarian, Irish, Italian, Latvian, Lithuanian, Maltese, Polish, Portuguese, Romanian, Slovak, Slovenian, Spanish and Swedish.'

Beyond this official statement, the EU actively encourages its citizens to learn other languages, both to facilitate professional and personal mobility within its single market and as a channel for cross-cultural contacts and mutual understanding. Learning and speaking other languages encourages people to become more open to others, their cultures and their outlooks; improves their cognitive skills and strengthens their ability to use their mother tongue, and enables people to reap the full benefits of the freedom to work or study in another Member State or outside the EU. 


\section{Do you think that there is at present a certain awareness on the part of the EU and DGT towards the non-official languages of the EU (e.g. Arabic, Chinese, African languages) with a greater use than some of the official languages?}

In our DG a certain (small) volume of texts from non-official EU languages is translated every year mainly by the English Department. Perhaps our Communication Unit can provide statistics on this matter if an official request is made.

Three of our 24 official languages are also official languages of the United Nations: English, French and Spanish. DGT holds regular meetings with the United Nations language services in the framework of IAMLADP and JIAMCATT to enhance exchanges among languages services of international organizations and national bodies.

In 2010, during the celebration of the European Year for combating poverty and social exclusion, the DGT held in Madrid, with the support of the team of professor Carmen Valero (University of Alcalá) and of the CEAR (Comisión Española de Ayuda al Refugiado), a seminar on Translation and Interpretation against social exclusion (the proceedings are available here: https://cvc.cervantes.es/lengua/tices/).

On the other hand, concerning the use of languages in Courts, the EU issued the Directive on the right to interpretation and translation in criminal proceedings and the European Commission supported the works of the Reflection Forum on Multilingualism and Interpreter Training, which drafted the background paper for implementation of EU Directive on the right to interpretation and translation in criminal proceedings.

Beyond the legislative framework, our DG has always been concerned about the situation of the profession in the Member States. In Spain, via our antenna in Madrid, we hosted and promoted the meetings of the RITAP (Network of Interpreters and Translators of the Spanish Public Administration) for the drafting of the White Paper on Institutional Translation and Interpreting in Spain, published thanks to the financial commitment of the European Commission and the Spanish Ministry of Foreign Affairs.

\section{Is IATE ready to integrate or expand into the most widely used non-official languages in the EU?}

That would not be a problem, at least from a technical point of view, since IATE can be fed with content in all languages, but the point is whether we have the capacity to manage content in non-EU languages for which we do not have translators or terminologists. We have to be realistic and concentrate on our priorities and mandate, which are complicated enough: management of a multilingual and multi-thematic database. We are very much aware that we cannot cover everything (every term in every domain) and we do not intend to become the only terminological resource in the world. The integration and the complementarity of terminological resources (for example throughout linked data applications) are the best solutions in the mid-term.

\section{How can IATE contribute to the dissemination or consolidation of Public Service} Interpreting and Translation (PSIT)? 
We are always willing to integrate validated and reliable terminological resources, especially those that add value to our documentary or phraseological resources (translation memories, aligned texts). Public Service Interpreting and Translation produces a good amount of terminological resources, which come from very different communicative situations and can therefore be very relevant for our work, which is not limited to legislative translation.

Last June our Department devoted the Seventh edition of our Institutional Terminology and Translation Day (a biennial event organised by the Spanish language Department with the support of the Commission Representation in Madrid) to the cooperation on terminology matters between Spanish universities and our Department. We presented to the CCDUTI (the body gathering all Departments of Translation and Interpretation in Spain) a draft of Memorandum of Understanding to channel this cooperation. According to this text, the main tasks and actions to be done are the following:

By the CCDUTI:

- provide a list of academic departments interested in collaborating with the Spanish Language Department (ES LD);

- draw up an inventory of the terminological work carried out by its members that may be useful for IATE;

- provide adequate training to their students in order to make them able to feed the IATE database.

By the ES LD:

- set up a list of thematic fields in which cooperation with CCDUTI should be a priority, given the lack of information on these fields in IATE;

- revision of the terminological contents from the CCDUTI to adapt them to the methodological criteria of the IATE database;

- dissemination (for example, in the interinstitutional bulletin puntoycoma, as well as in other networks in which the Department participates) of the main terminological works of the CCDUTI members.

Together, the CCDUTI and the ES LD, should explore:

- the specific ways of cooperation: traineeships at the DGT, creation of collaborative spaces through virtual networks, etc.;

- the methods of monitoring the project progress;

- the dissemination of the fruits of collaboration in forums and platforms such as Terminesp (when available) or in multilingual sites like Realiter.

\section{Since IATE is an open resource, how can the citizen, researcher, or trainer in non- EU languages contribute to the development and use of IATE?}

As a complement to the previous answer, terminological cooperation among different bodies is a give and take process with the ultimate goal of improving the dissemination of the appropriate terms and therefore, the validation of the terms resulting from its general acceptance. All actors should have the greatest interest in involving more qualified partners.

12. Any recommendations or advice for future translators who see the EU (or DGT) as a job opportunity? 
Apart from the job opportunities offered by the selection procedures organized by the institutions (see EPSO website, https://epso.europa.eu/home_en), increasing outsourcing at the DGT and also in other institutions opens up further opportunities for freelance translators.

Translation is a constantly changing and very demanding profession. A translator must be curious by nature. We advise our young colleagues to explore all the possibilities of their academic and professional horizons, without limiting themselves to a specific institution. 